\title{
The Application of Fluid Dynamics to Probability States in Superfluid Spacetime
}

\author{
Jerome Cantor ${ }^{1}$ \\ ${ }^{1}$ St John's University, Queens, NY, USA \\ Correspondence: Jerome Cantor, St John's University, Queens, NY, USA. E-mail: jocantor1@gmail.com
}

Received: February 6, 2018

Accepted: March 2, 2018

Online Published: March 21, 2018

doi:10.5539/apr.v10n2p21

URL: https://doi.org/10.5539/apr.v10n2p21

\begin{abstract}
The possibility that spacetime has the characteristics of a superfluid suggests that the relationship between velocity and time may be modeled in terms of percolation theory, where time dilation corresponds to increasing fluid viscosity. By equating superfluid percolation through a porous medium to conductance in a random resistor network, it was previously shown that the Lorentz factor corresponds to a probability function describing a phase transition to normal fluid. The current paper discusses how this novel linkage of momentum, time, and probability may provide a means of resolving conflicts between quantum mechanics and relativity theory.
\end{abstract}

Keywords: Lorentz factor, superfluid, spacetime, quantum mechanics

\section{Introduction}

A number of recent studies have suggested that spacetime may have the properties of superfluids, which exhibit quantum behavior at the macroscopic level, and may therefore provide a means of bridging the gap between quantum mechanics and relativity (Liberati \& Maccione, 2014; Volovik, 2013; Huang, Low, \& Roh-Suan, 2012; Bardeen, 1990). As previously shown by the author, the unique characteristics of superfluids may be incorporated into a model of spacetime that involves percolation processes, where complex macroscopic phenomena emerge from the random interaction of basic forces at lower levels of scale (Cantor, 2016). The concept is derived from studying the random action of fluid-like materials as they migrate through a latticework of channels. The distribution of connections between these channels determines the probability that they will become linked together in a network that permits the fluid to percolate from one end of the lattice to the other (Bunde \& Kantelhart, 2005). At a critical point in the evolution of the percolation system, a phase transition can occur, producing an entirely new pattern of physical or chemical behavior (Sahimi, 2009).

The percolation system that was previously used to develop a superfluid model of spacetime is the random resistor network, in which conducting bonds are indiscriminately disconnected (Cantor, 2016). In contrast to standard percolation systems, where porous channels are added, the loss of bonds results in increasing resistance to current flow, which eventually reaches a critical threshold involving a complete loss of conductivity (Redner, 2009). Using the decrease in conductance to model changes in superfluid density, it was previously shown that the probability of a random bond being disconnected may be expressed in terms of the Lorentz factor, suggesting that time dilation may correspond to a phase transition (Cantor, 2016).

Following a brief description of previous work that led to this finding, the current paper discusses the possibility that this linkage of time and velocity to probability may provide a theoretical construct for resolving certain conflicts between quantum mechanics and relativity theory.

\section{The Lorentz Factor as a Probability Function}

As shown by others, a 3-dimensional random resistor network may be used to model changes in fluid viscosity resulting from the loss of conductance (Farago \& Kantor, 2000; Huang, 1995; deGennes, 1976). We applied the features of this network to model time dilation in terms of a phase transition, and the primary equations involved in that process are reprised below (Cantor, 2016):

In a random-resistor network composed of uniform bonds, current density $(j)$ may be expressed as:

$$
j=\rho_{s} v_{s}+\rho_{n} v_{n}
$$


where $\rho_{s} v_{s}$ and $\rho_{n} v_{n}$ are the density $(\rho)$ and velocity $(v)$ of the superfluid and normal fluid components, respectively, and $p_{s}+p_{n}$ is constant (Huang, 1995).

If the network channels are sufficiently small, $v_{n}=0$, allowing the normal fluid component to be removed from the equation. Thus,

$$
j=\rho_{s} \nabla \Phi
$$

where $\Phi$ represents the electrostatic potential or pressure differential across the network.

Using Poiseuille's law (Sutera \& Skalak, 1993), the cross-sectional area (CSA) of the network bonds may be related to the pressure differential,

$$
\frac{A_{x}}{A_{t}}\left(\frac{\rho_{n x}}{\rho_{n t}}\right)=\frac{P_{1 x}-P_{2 x}}{P_{1 t}-P_{2 t}}
$$

where $A_{x}$ is the CSA of the disconnected bonds at a given point, $\rho_{n x}$ is normal fluid density at the same point, $A_{t}$ is the total CSA of the network bonds, $\rho_{n t}$ is the maximal normal fluid density, $P_{1 x}-P_{2 x}$ is the pressure differential at the given point, and $P_{1 t}-P_{2 t}$ is the maximal pressure differential.

The relative CSA of non-conducting bonds is a measure of the probability $(p)$ that a random bond is disconnected, and an equivalent probability is applicable to the relative density of normal fluid, which also depends on CSA of disconnected bonds. Thus,

$$
p=\frac{A_{x}}{A_{t}}=\frac{\rho_{n x}}{\rho_{n t}}
$$

In accordance with Bernoulli's principle, an inertial frame moving at different velocities through fluid spacetime of constant density exhibits pressure changes based on the square of its velocity. Equation 3 may therefore be expressed in terms of probability and velocity:

$$
p^{2}=\frac{c^{2}-v^{2}}{c^{2}}
$$

Or,

$$
p=\sqrt{1-\frac{v^{2}}{c^{2}}}
$$

\section{The Relationship between Probability Density and Level of Scale}

The linkage of velocity, time, and probability, shown in equation 6 , offers a new approach to characterizing uncertainty for quantum scale interactions. The probability that a conducting bond is disconnected would have increasing variability at decreasing levels of scale, and the movement of time would become discontinuous as the number of bonds used to locally determine $p$ approaches zero (Figure 1). Similarly, velocity (and therefore momentum) becomes discontinuous and subject to increasing uncertainty.

For a specified frame velocity, the level of uncertainty may be expressed as the probability $(\alpha)$ that a given group of bonds has the ratio of disconnected to total bond CSA predicted by the Lorentz factor, as shown in the probability density function below:

$$
\int_{-\infty}^{\infty} F \alpha(x) d x=1
$$

with $x$ equivalent to:

$$
\frac{A x p}{A t}-\frac{A x a}{A t}
$$

where $\frac{A_{x p}}{A_{t}}$ is the predicted ratio of disconnected to total bond CSA and $\frac{A_{x a}}{A_{t}}$ is the actual ratio, which may also be expressed as:

$$
\frac{\sum_{1}^{\infty} p_{1}+p_{2}+p_{3} \ldots p_{n}}{n}
$$

where $p$ is the probability that random bonds within a uniform subdivision of the network are disconnected. 


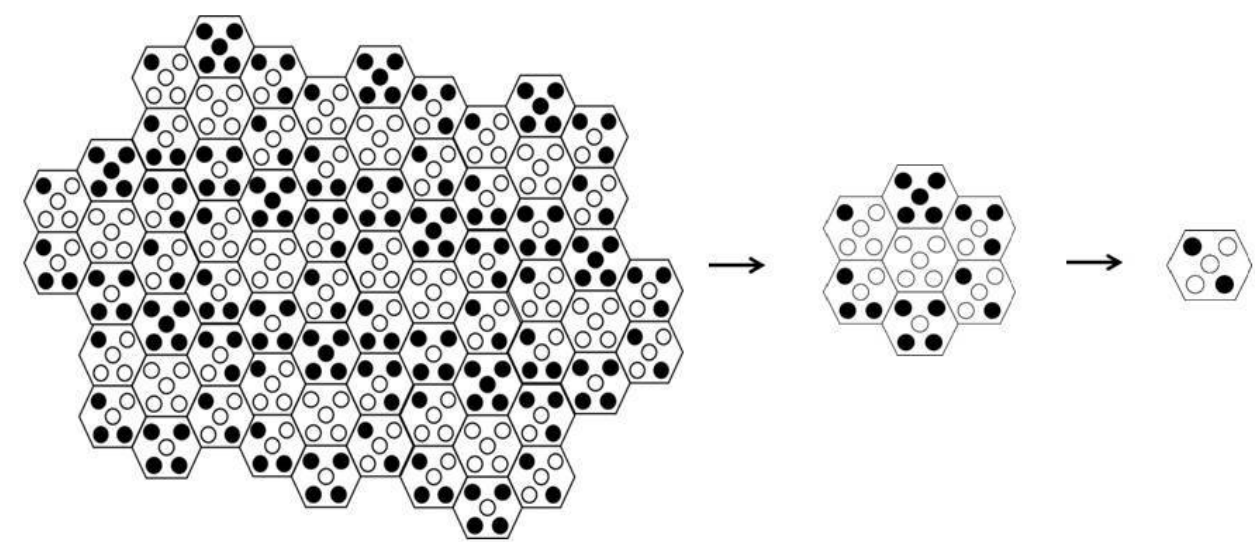

Figure 1. As the number of intact (white) and disconnected (black) bonds used to determine $p$ approaches zero, both time and momentum become discontinuous

At increasing levels of scale, $\alpha$ approaches zero, and the probability curve begins to converge, producing a normal distribution with an infinitely narrow spike. This decrease in $\alpha$ would be associated with the appearance of continuity in both momentum and time (Figure 2).

\section{Potential Limitations of the Model}

It may be argued that the Lorentz factor expresses a precise relationship between time and motion, and is not an appropriate measure of probability. However, the use of this factor as a determinant of probability only becomes meaningful at the lowest levels of scale, where partitioning the network into increasingly smaller segments results in a virtually random positioning of open and closed bonds (Figure 2). At this level, time and velocity are governed by a process resembling that of throwing dice, thus providing a rationale for the expression of probabilities in terms of the Lorentz factor.

Nevertheless, one could argue that these probabilities cannot be separated from the fixed properties of the network, where (to pursue the analogy) the dice have already been thrown. While this may be the case at higher levels of scale, it should not affect measurements of quantum events, where the degree of uncertainty would be similar to that of unstructured activity.

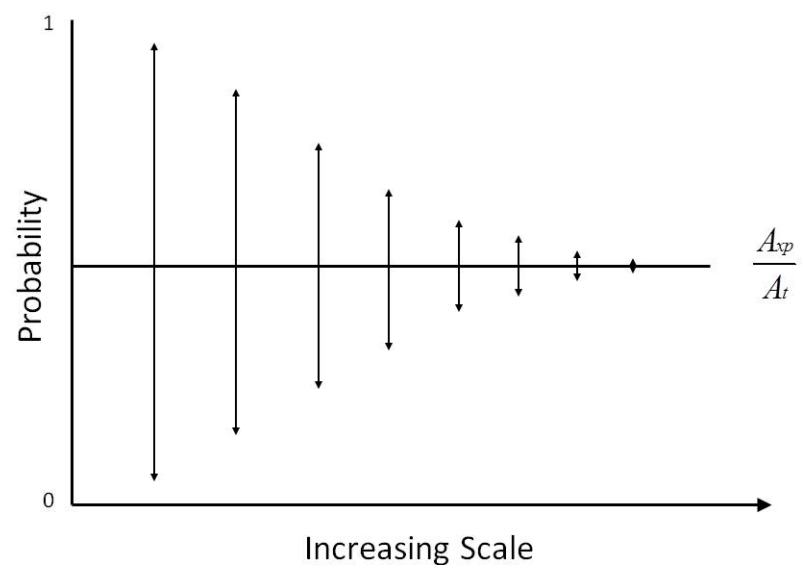

Figure 2. The probability that a random network bond is disconnected converges at increasing levels of scale,

$$
\text { where } \frac{A_{x p}}{A_{t}} \text { is the predicted value }
$$

\section{Other Approaches}

While the application of fluid dynamics is an essential component of the current work, other theoretical approaches also support probabilistic modeling of phase transitions. For various media, the critical exponent involved in modeling such processes is $1 / 2$, and may be expressed as: 


$$
\left|\frac{p_{c}-p}{p_{c}}\right|^{1 / 2} p_{c}-p \ll 1
$$

where $p_{c}$ is the critical probability at the transition point (Papon, Leblond, \& Meijer, 2006; Annett, 2004). This equation may also be used to model superfluid conductance $(G)$ in a random resistor network:

$$
G \propto\left|1-\frac{p}{p_{c}}\right|^{1 / 2}
$$

where substitution of $v^{2}$ for $p$ and $c^{2}$ for $p_{c}$ yields the Lorentz factor.

Another approach involves the movement of electrons through a 3-dimensional lattice, where there is loss of Anderson localization with increasing scale, as follows:

$$
g(L)=\sigma L^{d-2}
$$

where $g$ represents dimensionless conductance, $L$ is the size of the network, $d$ is the network dimension, and $\sigma$ is conductivity (Lee \& Ramakrishnan, 1985). As the scale increases, conductivity moves away from disorder, and becomes consistent with Ohm's law, which is analogous to the appearance of continuity in the current model (Chandrashekar \& Busch, 2014; Lee \& Ramakrishnan, 1985).

\section{Conclusions}

The novel use of a random resistor network to model superfluid spacetime may provide a framework for resolving the dichotomy between relativity theory and quantum mechanics. The transition from wave-like properties of matter to particle-based characteristics is a natural consequence of expressing the Lorentz factor in terms of a probability function. The difference between the two states of matter thus becomes a question of scale, and the convergence of the probability density function shown in equation 7 may account for the transition from wave to particle behavior. Whether this theoretical construct can provide a new understanding of the physical universe will require further investigation of the potential relationship between superfluid dynamics and the structure of spacetime.

\section{References}

Annett, J. (2004). Superconductivity, superfluids and condensates (pp 21-46). New York, USA: Oxford University Press. https://doi.org/10.1016/j.materresbull.2005.03.010

Bardeen, J. (1990). Superconductivity and other macroscopic quantum phenomena. Physics Today, 43, 25-31. https://doi.org/10.1063/1.881218.

Bunde, A., \& Kantelhart, J. W. (2005). Diffusion and conduction in percolation systems. In P. Heitjans, \& J. Karger (Eds.), Diffusion in condensed matter (pp. 895-914). Berlin: Springer.

Cantor, J. (2016). Is the Lorentz factor a probability function in superfluid spacetime? App. Phys. Res., 8, 38-41. https://doi.org/10.5539/apr.v8n3p1

Chandrashekar, C. M., \& Busch, Th. (2014). Quantum percolation and transition point of a directed discrete-time quantum walk. Sci. Rep., 4, 6583. https://doi.org/10.1038/srep06583

deGennes, P. G. (1976). On a relation between percolation theory and the elasticity of gels. J. Physique Lett., 37, L1-L3. https://doi.org/10.1051/jphyslet:019760037010100

Farago, O., \& Kantor, Y. (2000). Entropic elasticity of phantom percolation networks. Europhys. Lett., 52, 413419. https://doi.org/10.1209/epl/i2000-00453-y

Huang, K. (1995). Bose-Einstein condensation and superfluidity. In A. Griffin, D. W. Snoke, \& S. Stringari (Eds.), Bose-Einstein condensation (pp. 31-50). Cambridge, England: Cambridge University Press.

Huang, K., Low, H.-B., \& Roh-Suan, T. (2012). Scalar field cosmology: I. Asymptotic freedom and the initialvalue problem. Class. Quantum Grav., 29, 155014. https://doi.org/10.1088/0264-9381/29/15/155014

Lee, P. A., \& Ramakrishnan, T. V. (1985). Disordered electronic systems. Rev. Mod. Phys., 57, 287-337. https://doi.org/10.1103/RevModPhys.57.287

Liberati, S., \& Maccione, L. (2014). Astrophysical constraints on Planck scale dissipative phenomena. Phys. Rev. Lett., 112, 151301. https://doi.org/10.1103/PhysRevLett.112.151301 
Papon, P., Leblond, J., \& Meijer, P. H. E. (2006). The physics of phase transitions: concepts and applications (2nd ed., pp. 215-250). Berlin, Germany: Springer.

Redner, S. (2009) Fractal and multifractal scaling of electrical conduction in random resistor networks. In P. A. Meyers (Ed.), Encyclopedia of complexity and systems science (pp. 3737-3754). Berlin: Springer.

Sahimi, M. (2009) Percolation phase transition. In P. A. Meyers (Ed.), Encyclopedia of complexity and systems science (pp. 6538-6545). Berlin: Springer.

Sutera S. P., \& Skalak R. (1993). The history of Poiseuille's law. Annu. Rev. Fluid Mech., 25, 1-19. https://doi.org/10.1146/annurev.fl.25.010193.000245

Volovik, G. E. (2013). The superfluid universe. In K. H. Bennemann, \& J. B. Ketterson (Eds.), Novel superfluids (Vol. 1, pp. 570-618). Oxford: Oxford Univ. Press.

\section{Copyrights}

Copyright for this article is retained by the author(s), with first publication rights granted to the journal.

This is an open-access article distributed under the terms and conditions of the Creative Commons Attribution license (http://creativecommons.org/licenses/by/4.0/). 